\title{
Beam energy and system dependence of rapidity-even dipolar flow
}

\author{
Niseem Magdy (For the STAR Collaboration) ${ }^{1 \star}$ \\ ${ }^{1}$ Department of Chemistry, Stony Brook University, Stony Brook, NY, 11794-3400, USA
}

\begin{abstract}
New measurements of rapidity-even dipolar flow, $\mathrm{v}_{1}^{\text {even }}$, are presented for several transverse momenta, $p_{T}$, and centrality intervals in $\mathrm{Au}+\mathrm{Au}$ collisions at $\sqrt{s_{N N}}=200,39$ and $19.6 \mathrm{GeV}, \mathrm{U}+\mathrm{U}$ collisions at $\sqrt{s_{N N}}=193 \mathrm{GeV}$, and $\mathrm{Cu}+\mathrm{Au}$, $\mathrm{Cu}+\mathrm{Cu}, \mathrm{d}+\mathrm{Au}$ and $\mathrm{p}+\mathrm{Au}$ collisions at $\sqrt{s_{N N}}=200 \mathrm{GeV}$. The $\mathrm{v}_{1}^{\text {even }}$ shows characteristic dependencies on $p_{T}$, centrality, collision system and $\sqrt{s_{N N}}$, consistent with the expectation from a hydrodynamic-like expansion to the dipolar fluctuation in the initial state. These measurements could serve as constraints to distinguish between different initialstate models, and aid a more reliable extraction of the specific viscosity $\eta / s$.
\end{abstract}

\section{Introduction}

Heavy-ion collisions (HIC) at the Relativistic Heavy Ion Collider (RHIC) and the Large Hadron Collider (LHC) are aimed at studying the properties of the strongly interacting quark-gluon plasma (QGP) created in such collisions. Recent studies have emphasized the use of anisotropic flow measurements to study the transport properties of the QGP [1-7]. A crucial question in these studies was the role of initial-state fluctuations and their influence on the uncertainties associated with the extraction of $\eta / s$ for the QGP produced in HIC $[8,9]$. This work emphasizes new measurements for rapidity-even dipolar flow, $v_{1}^{\text {even }}$, which could aid a distinction between different initial-state models and facilitate the extraction of $\eta / s$ with better constraints.

Anisotropic flow is characterized by the Fourier coefficients, $\mathrm{v}_{n}$, obtained from a Fourier expansion of the azimuthal angle $(\phi)$ distribution of the emitted particles [10]:

$$
\frac{d N}{d \phi} \propto 1+2 \sum_{n=1} \mathrm{v}_{\mathrm{n}} \cos \left(n\left(\phi-\Psi_{n}\right)\right)
$$

where $\Psi_{n}$ represents the $n^{\text {th }}$-order event plane, the coefficients $\mathrm{v}_{1}, \mathrm{v}_{2}$ and $\mathrm{v}_{3}$ are called directed, elliptic and triangular flow, respectively. The flow coefficients $\mathrm{v}_{n}$ are related to the two-particle Fourier coefficients $\mathrm{v}_{n, n}$ as:

$$
\mathrm{v}_{\mathrm{n}, \mathrm{n}}\left(p_{\mathrm{T}}^{a}, p_{\mathrm{T}}^{b}\right)=\mathrm{v}_{\mathrm{n}}\left(p_{\mathrm{T}}^{a}\right) \mathrm{v}_{\mathrm{n}}\left(p_{\mathrm{T}}^{b}\right)+\delta_{N F},
$$

where $p_{\mathrm{T}}^{a}$ and $p_{\mathrm{T}}^{b}$ are the transvers momentum of particles (a) and (b), respectively, and $\delta_{N F}$ is a so-called non-flow (NF) term, which includes possible contributions from resonance decays, BoseEinstein correlations, jets, and global momentum conservation (GMC) [11-15]. The directed flow, $\mathrm{v}_{1}$,

\footnotetext{
^e-mail: niseemm@gmail.com
} 


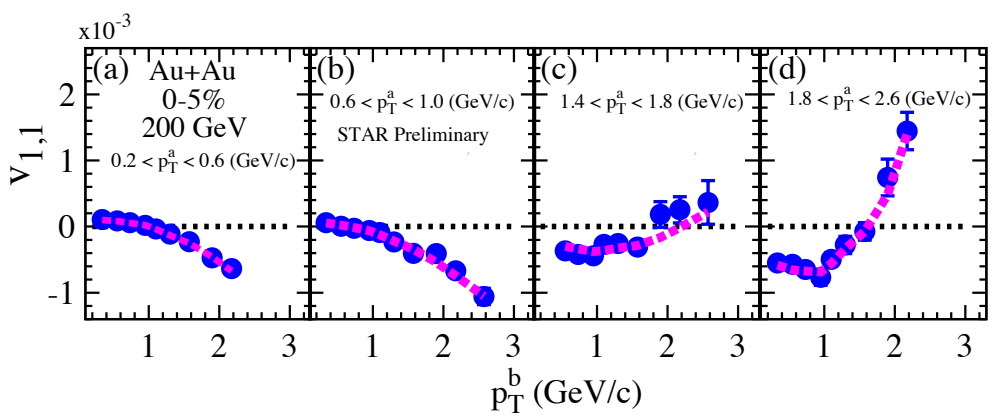

Figure 1. $\mathrm{v}_{1,1}$ vs. $p_{T}^{b}$ for several selections of $p_{T}^{a}$ for $0-5 \%$ central $\mathrm{Au}+\mathrm{Au}$ collisions at $\sqrt{s_{N N}}=200 \mathrm{GeV}$. The dashed curve shows the result of the simultaneous fit with Eq. 5.

can be separated into an odd function of pseudorapidity $(\eta)$ [16] which develops along the direction of the impact parameter, and a rapidity-even component $[13,17]$ which results from the effects of initialstate fluctuations acting in concert with a hydrodynamic-like expansion; $\mathrm{v}_{1}(\eta)=\mathrm{v}_{1}^{\text {even }}(\eta)+\mathrm{v}_{1}^{\text {odd }}(\eta)$, where $\Psi_{1}^{\text {odd }}$ and $\Psi_{1}^{\text {even }}$ are uncorrelated. The magnitude of $\mathrm{v}_{1}^{\text {even }}$ is related to the fluctuations-driven dipole asymmetry $\varepsilon_{1}$ and $\eta / s[14,17,18]$.

\section{Measurements}

The correlation function technique was used to generate the two-particle $\Delta \phi$ correlations:

$$
C_{r}(\Delta \phi, \Delta \eta)=\frac{(d N / d \Delta \phi)_{\text {same }}}{(d N / d \Delta \phi)_{\text {mixed }}},
$$

where $(d N / d \Delta \phi)_{\text {same }}$ represent the normalized azimuthal distribution of particle pairs from the same event and $(d N / d \Delta \phi)_{\text {mixed }}$ represents the normalized azimuthal distribution for particle pairs in which each member is selected from a different event but with a similar classification for the vertex, centrality, etc. The pseudorapidity requirement $|\Delta \eta|>0.7$ was also imposed on track pairs to minimize possible non-flow contributions associated with the short-range correlations from resonance decays, Bose-Einstein correlations and jets.

The two-particle Fourier coefficients $\mathrm{v}_{n, n}$ are obtained from the correlation function as:

$$
\mathrm{v}_{\mathrm{n}, \mathrm{n}}=\frac{\sum_{\Delta \phi} C_{r}(\Delta \phi, \Delta \eta) \cos (n \Delta \phi)}{\sum_{\Delta \phi} C_{r}(\Delta \phi, \Delta \eta)},
$$

and then used to extract $v_{1}^{\text {even }}$ via a simultaneous fit of $\mathrm{v}_{1,1}$ as a function of $p_{T}^{\mathrm{b}}$, for several selections of $p_{T}^{a}$ with Eq. 2:

$$
\mathrm{v}_{1,1}\left(p_{\mathrm{T}}^{a}, p_{\mathrm{T}}^{b}\right)=\mathrm{v}_{1}^{\text {even }}\left(p_{\mathrm{T}}^{a}\right) \mathrm{v}_{1}^{\text {even }}\left(p_{\mathrm{T}}^{b}\right)-C p_{\mathrm{T}}^{a} p_{\mathrm{T}}^{b} .
$$

Here, $C \propto 1 /\left(\langle M u l t\rangle\left\langle p_{T}^{2}\right\rangle\right)$ takes into account the non-flow correlations induced by a global momentum conservation $[14,15]$ and $\langle$ Mult $\rangle$ is the mean multiplicity.

For a given centrality selection, the left hand side of Eq. 5 represents the $N \times N$ matrix which we fit with the right hand side using $N+1$ parameters; $\mathrm{N}$ values of $\mathrm{v}_{1}^{\text {even }}\left(p_{\mathrm{T}}\right)$ and one additional parameter $C$, accounting for momentum conservation [19]. Fig. 1 shows a representative result for this fitting procedure for $0-5 \%$ central $\mathrm{Au}+\mathrm{Au}$ collisions at $\sqrt{s_{N N}}=200 \mathrm{GeV}$. The dashed curve (obtained with Eq. 5) in each panel illustrates the effectiveness of the simultaneous fits, as well as the constraining power of the data. That is, $\mathrm{v}_{1,1}\left(p_{\mathrm{T}}^{b}\right)$ evolves from negative to positive values as the selection range for $p_{\mathrm{T}}^{a}$ is increased. 


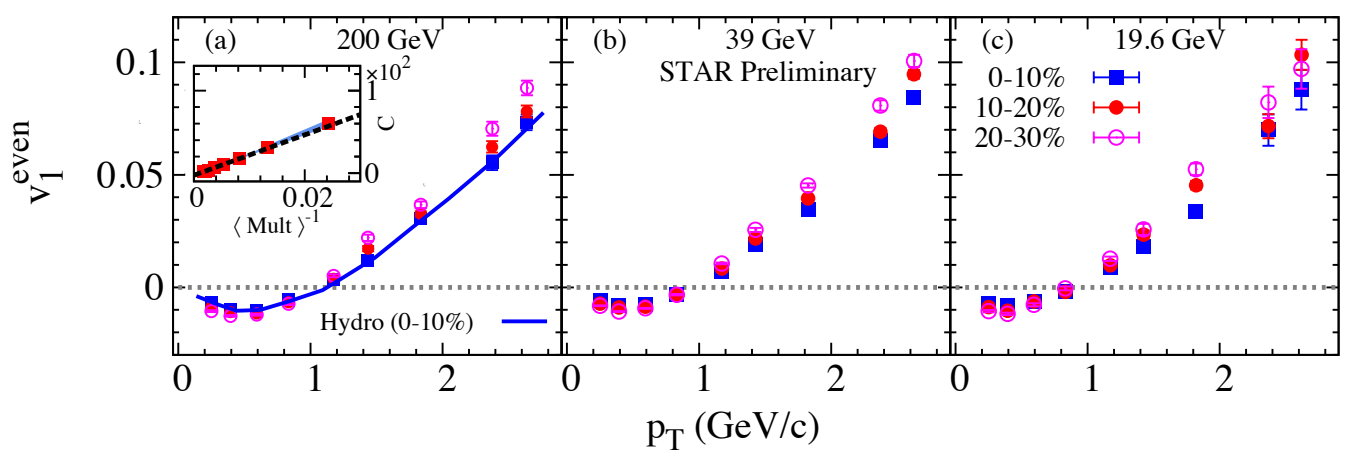

Figure 2. Extracted values of $v_{1}^{\text {even }}$ vs. $p_{T}$ for different centrality selections $(0-10 \%, 10-20 \%$ and $20-30 \%) \mathrm{Au}+\mathrm{Au}$ collisions for several values of $\sqrt{S_{N N}}$ as indicated; the $v_{1}^{\text {even }}$ values are obtained via fits with Eq. (5). The solid line in panel (a) shows the result from a hydrodynamic calculations with $\eta / s=0.16$ [14]. The inset in panel (a) shows a representative set of the associated values of $C$ vs. $\langle M u l t\rangle^{-1}$.

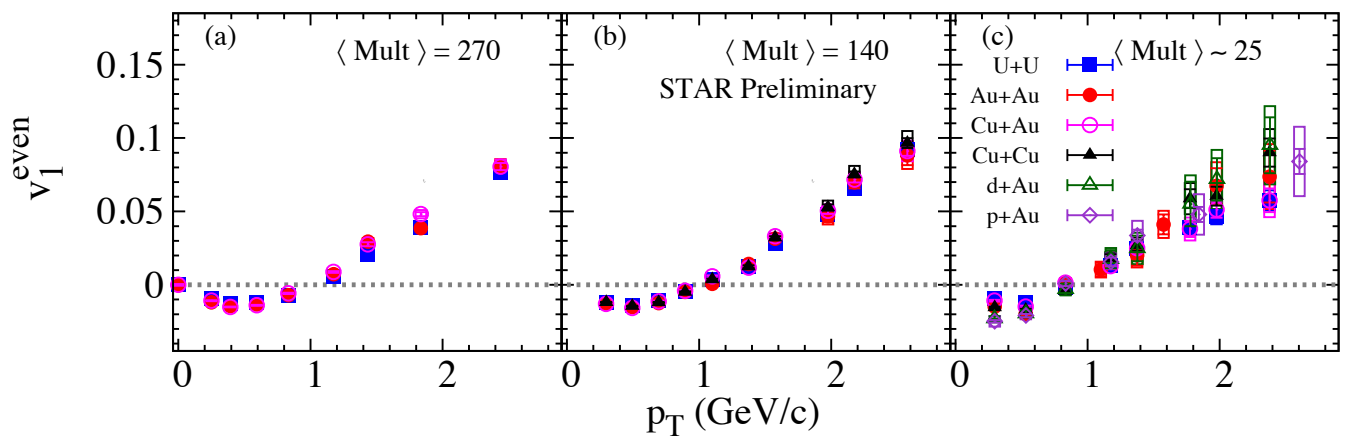

Figure 3. Extracted values of $v_{1}^{\text {even }}$ vs. $p_{T}$ for different $\langle$ Mult $\rangle$ selections for different collisions system at $\sqrt{s_{N N}} \sim 200 \mathrm{GeV}$ as indicated; the $\mathrm{v}_{1}^{\text {even }}$ values are obtained via fits with Eq. (5).

\section{Results}

Representative $v_{1}^{\text {even }}$ results for $\mathrm{Au}+\mathrm{Au}$ collisions at $\sqrt{s_{N N}}=200,39$, and $19.6 \mathrm{GeV}$ and for different collision systems $\mathrm{U}+\mathrm{U}$ at $\sqrt{s_{N N}}=193 \mathrm{GeV}$, and $\mathrm{Cu}+\mathrm{Au}, \mathrm{Cu}+\mathrm{Cu}, \mathrm{d}+\mathrm{Au}$ and $\mathrm{p}+\mathrm{Au}$ at $\sqrt{s_{N N}}=200$ $\mathrm{GeV}$ are summarized in Figs. 2 and 3. The values of $v_{1}^{e v e n}\left(p_{\mathrm{T}}\right)$ extacted for different centrality selections $(0-10 \%, 10-20 \%$ and $20-30 \%)$ are shown in Fig. 2; the solid line in panel (a) shows the a hydrodynamic calculations with $\eta / s=0.16[14]$, which in good agreement with our measurements, the inset shows the corresponding results for the associated momentum conservation coefficient, $C$, extracted for several centralities at $\sqrt{s_{N N}}=200 \mathrm{GeV}$. The $v_{1}^{\text {even }}\left(p_{\mathrm{T}}\right)$ values indicate the characteristic pattern of a change from negative $v_{1}^{\text {even }}\left(p_{\mathrm{T}}\right)$ at low $p_{\mathrm{T}}$ to positive $\mathrm{v}_{1}^{\text {even }}\left(p_{\mathrm{T}}\right)$ for $p_{\mathrm{T}}>1 \mathrm{GeV} / \mathrm{c}$, with a crossing point that shifts with $\sqrt{s_{N N}}$. They also indicate that $v_{1}^{\text {even }}$ increase as the centrality become more peripheral, as might be expected from the centrality dependence of $\varepsilon_{1}$.

The extracted values of $v_{1}^{\text {even }}\left(p_{\mathrm{T}}\right)$, for different collision systems are compared in Fig. 3 for different values of $\langle$ Mult $\rangle$. Figs. 3(a), 3(b) and 3(c) indicate similar $v_{1}^{\text {even }}\left(p_{\mathrm{T}}\right)$ magnitudes for the systems specified at each $\langle$ Mult $\rangle$, as well as the characteristic pattern of a change from negative $v_{1}^{\text {even }}\left(p_{\mathrm{T}}\right)$ at low $p_{\mathrm{T}}$ to positive $\mathrm{v}_{1}^{\text {even }}\left(p_{\mathrm{T}}\right)$ for $p_{\mathrm{T}}>1 \mathrm{GeV}$. This pattern confirms the predicted trends for rapidityeven dipolar flow $[13,14,17]$ and further indicates that for the selected values of $\langle M u l t\rangle, v_{1}^{\text {even }}\left(p_{\mathrm{T}}\right)$ does not show a strong dependence on the collision system. This apparent system independence of $\mathrm{v}_{1}^{\text {even }}\left(p_{\mathrm{T}}\right)$ for the indicated $\langle$ Mult $\rangle$ values suggests that the fluctuations-driven initial-state eccentricity 
$\varepsilon_{1}$, is similar for the six collision systems. It also suggests that the viscous effects that are related to $\eta / s$ are comparable for the matter created in each of these collision systems.

\section{Conclusion}

In summary, we have used the two-particle correlation method to carry out new differential measurements of rapidity-even dipolar flow, $\mathrm{v}_{1}^{\text {even }}$, in $\mathrm{Au}+\mathrm{Au}$ collisions at different beam energies, and in $\mathrm{U}+\mathrm{U}, \mathrm{Cu}+\mathrm{Au}, \mathrm{Cu}+\mathrm{Cu}, \mathrm{d}+\mathrm{Au}$ and $\mathrm{p}+\mathrm{Au}$ collisions at $\sqrt{s_{N N}} \simeq 200 \mathrm{GeV}$. The measurements confirm the characteristic patterns of an evolution from negative $v_{1}^{\text {even }}\left(p_{\mathrm{T}}\right)$ for $p_{\mathrm{T}}>1 \mathrm{GeV} / \mathrm{c}$ to positive $\mathrm{v}_{1}^{\text {even }}\left(p_{\mathrm{T}}\right)$ for $p_{\mathrm{T}}>1 \mathrm{GeV} / \mathrm{c}$, expected when initial-state geometric fluctuations act in concert with the hydrodynamic-like expansion to generate rapidity-even dipolar flow. This measurements provide additional constraints which are important to discern between different initial-state models, and to aid precision extraction of the temperature dependence of the specific shear viscosity.

\section{Acknowledgments}

This research is supported by the US Department of Energy under contract DE-FG0287ER40331.A008.

\section{References}

[1] D. Teaney, Phys.Rev. C68, 034913 (2003), nucl-th/0301099

[2] R.A. Lacey, A. Taranenko, PoS CFRNC2006, 021 (2006), nucl-ex/0610029

[3] B. Schenke, S. Jeon, C. Gale, Phys.Lett. B702, 59 (2011), 1102.0575

[4] H. Song, S.A. Bass, U. Heinz, Phys.Rev. C83, 054912 (2011), 1103.2380

[5] H. Niemi, G. Denicol, P. Huovinen, E. Molnar, D. Rischke, Phys.Rev. C86, 014909 (2012), 1203.2452

[6] G.Y. Qin, H. Petersen, S.A. Bass, B. Muller, Phys.Rev. C82, 064903 (2010), 1009. 1847

[7] N. Magdy (STAR), J. Phys. Conf. Ser. 779, 012060 (2017)

[8] B. Alver, G. Roland, Phys. Rev. C81, 054905 (2010), [Erratum: Phys. Rev.C82,039903(2010)], 1003.0194

[9] R.A. Lacey, D. Reynolds, A. Taranenko, N.N. Ajitanand, J.M. Alexander, F.H. Liu, Y. Gu, A. Mwai, J. Phys. G43, 10LT01 (2016), 1311.1728

[10] A.M. Poskanzer, S.A. Voloshin, Phys. Rev. C58, 1671 (1998), nucl-ex/9805001

[11] R.A. Lacey, Nucl. Phys. A774, 199 (2006), nucl-ex/0510029

[12] N. Borghini, P.M. Dinh, J.Y. Ollitrault, Phys. Rev. C62, 034902 (2000), nucl-th/0004026

[13] M. Luzum, J.Y. Ollitrault, Phys. Rev. Lett. 106, 102301 (2011), 1011.6361

[14] E. Retinskaya, M. Luzum, J.Y. Ollitrault, Phys. Rev. Lett. 108, 252302 (2012), 1203.0931

[15] G. Aad et al. (ATLAS), Phys. Rev. C86, 014907 (2012), 1203. 3087

[16] P. Danielewicz, R. Lacey, W.G. Lynch, Science 298, 1592 (2002), nucl-th/0208016

[17] D. Teaney, L. Yan, Phys. Rev. C83, 064904 (2011), 1010. 1876

[18] F.G. Gardim, F. Grassi, Y. Hama, M. Luzum, J.Y. Ollitrault, Phys. Rev. C83, 064901 (2011), 1103.4605

[19] J. Jia, S.K. Radhakrishnan, S. Mohapatra, J. Phys. G40, 105108 (2013), 1203 . 3410 